\title{
Coronary vessel wall assessment after Kawasaki Disease
}

\author{
Tarique Hussain*, Sarah Peel, Aphrodite Tzifa, Thomas Krasemann, Gerald Greil, Rene Botnar \\ From 2011 SCMR/Euro CMR Joint Scientific Sessions \\ Nice, France. 3-6 February 2011
}

\section{Introduction}

2D cross-sectional Dual-Inversion-Recovery (DIR) blackblood imaging of the coronary arteries has been used to assess Kawasaki Disease (KD) (Greil et al. 2007). A 3D coronary black-blood approach, however, has been shown to provide better coverage of the coronary tree (Botnar et al 2001). Furthermore, uptake of gadolinium contrast agent in coronary walls may indicate on-going inflammation or vessel wall fibrosis (Maintz et al, 2006).

\section{Aim}

To investigate whether in-plane 3D coronary vessel wall imaging utilizing a local-inversion technique and spiral image acquisition, in combination with contrastenhanced inversion-recovery (IR) imaging allows more comprehensive assessment of KD.

\section{Methods}

Patients with previous coronary aneurysms due to KD undergoing routine CMR evaluation had additional vessel wall imaging before and after contrast administration $(0.2 \mathrm{mmol} / \mathrm{kg}$ gadopentetate dimeglumine). All examinations were performed on a $1.5 \mathrm{~T}$ MR-system and the coil was selected according to patient size (two-element/fiveelement). Coronary MRA was followed by a targeted, free-breathing, ECG-triggered 3D vessel wall sequence using local inversion and spiral image acquisition (spatial resolution $=0.76 \times 0.76 \times 2 \mathrm{~mm} ; \mathrm{TE} / \mathrm{TR}=2 / 29 \mathrm{~ms}$, $\mathrm{FA}=90^{\circ}$ ). Areas of vessel wall thickening on in-plane imaging were then further evaluated with through plane 2D DIR vessel wall technique.

Post-contrast imaging was performed using a freebreathing, ECG-triggered, 3D IR segmented gradientecho (TFE) sequence (spatial resolution $=1.25 \times 1.25 \times 3 \mathrm{~mm}$,

King's College London, London, UK
$\mathrm{TE} / \mathrm{TR}=1.4 / 3.5 \mathrm{~ms}, \mathrm{FA}=30^{\circ} \& \mathrm{TI}$ chosen to null blood using Look-Locker).

\section{Results}

13 CMR examinations were performed in 12 children (7 male, age $=2-19 \mathrm{yrs} ; \mathrm{HR}=60-110 \mathrm{bpm} ; 6$ weeks -18 years post-acute KD). 6 cases showed persistent aneurysms; 4 cases had coronary ectasia and the remaining resolved aneurysms had a normal lumen. One case showed a mild LAD stenosis shown on CMRA and confirmed on conventional angiography. Complete black blood coverage of the affected portions of the coronary arteries was achieved using the new 3D in-plane local-inversion technique. Targeted 2D cross-sectional imaging showed that aneurismal segments had greater wall thickness

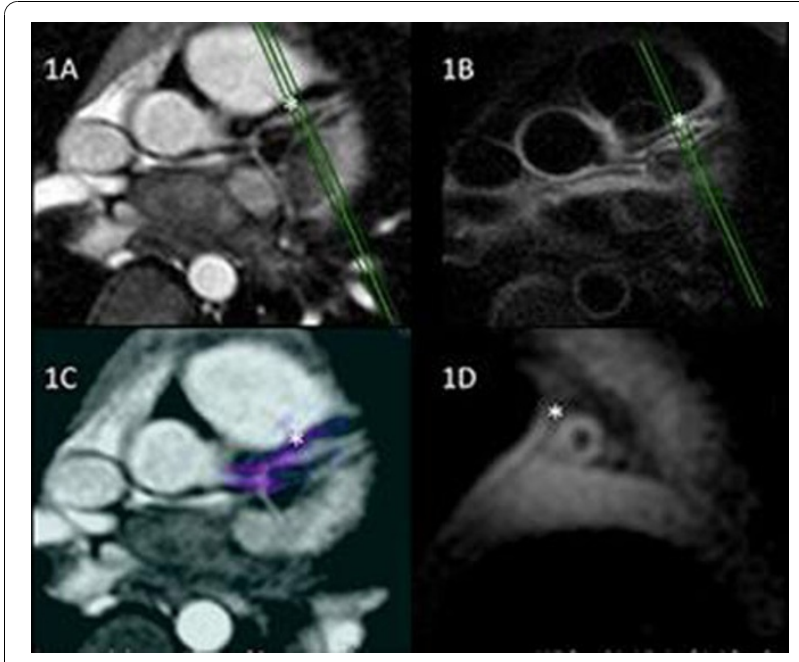

Figure 1 4yrs old patient ( 9 months post-acute KD). CMRA (1A) showing LAD aneurysm $\left(^{*}\right)$. 3D in-plane local inversion black blood of LAD (1B). Delayed Enhancement overlay (purple) on CMRA showing enhancement of aneurysm (1C). Green marker on 1A \& 1B shows position of DIR cross-section (1D). 


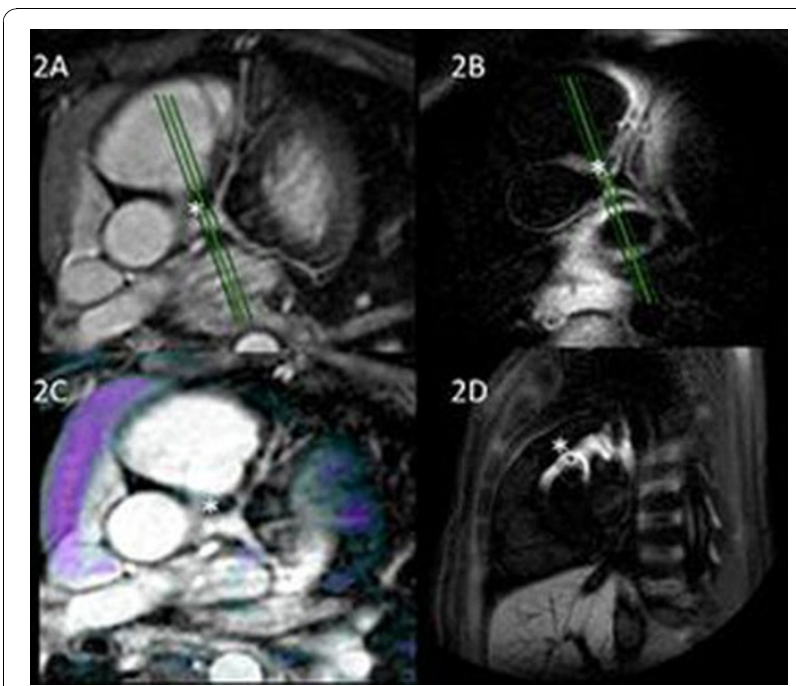

Figure 2 4yrs old patient (3 yrs post-acute KD). CMRA (2A) showing Left Main extasia (*). 3D in-plane local inversion black blood of left main (2B). Delayed Enhancement overlay (purple) on CMRA showing no persisting enhancement (2C). Green marker on 2A \& 2B shows position of DIR cross-section (2D).

(mean $0.87 \mathrm{~mm})$ than ectatic segments $(0.42 \mathrm{~mm})$ or resolved aneurysms $(0.31 \mathrm{~mm})$. Delayed enhancement of the coronary vessel wall was only present in three cases (all of whom had recent KD $<2$ yrs ago). Figures 1 and 2.

\section{Conclusion}

Our data suggests that inflammation of the coronary vessel wall in Kawasaki disease may persist for up to 2 years post-acute insult. Vessel wall remodeling seems to have occurred effectively in the resolved aneurysms studied here. 3D in-plane local-inversion black-blood imaging gives more complete coverage of affected segments and allows targeting of cross-sectional imaging of thickened areas. The protocol outlined here gives a more comprehensive assessment of affected coronaries after $\mathrm{KD}$.

Published: 2 February 2011

doi:10.1186/1532-429X-13-S1-P235

Cite this article as: Hussain et al: Coronary vessel wall assessment after Kawasaki Disease. Journal of Cardiovascular Magnetic Resonance 201113 (Suppl 1):P235,

\section{Submit your next manuscript to BioMed Central} and take full advantage of:

- Convenient online submission

- Thorough peer review

- No space constraints or color figure charges

- Immediate publication on acceptance

- Inclusion in PubMed, CAS, Scopus and Google Scholar

- Research which is freely available for redistribution
Biomed Central 